\title{
ILUSTRACIÓN, ROMANTICISMO, POSTMODERNIDAD: EL TIEMPO EN LA LITERATURA INGLESA
}

\section{Michael J. Gronow}

Para poder insertar este estudio dentro del campo de la historia cultural, nos basamos en la definición de la misma ofrecida por Lynn Hunt en "History, Culture, and Text" donde dicho historiador explora el concepto en cuestión en términos de la interacción entre fenómenos específicos y tendencias generales dentro de un periodo histórico determinado, un proceso que, además, implica la interrelación de estructura y evento, contribuyendo así a resaltar la significación cultural como un texto que puede leerse (1989: 12-13). Como la propia referencia al término 'texto' indica, se solapará en todo momento el marco de la historia cultural ya mencionado con otro relacionado con la teoría literaria. En este sentido, desde una perspectiva post-estructuralista como la que ofrece Fredric Jameson, en cuanto a la historia cultural como texto se refiere, se pretende trazar el gráfico de cómo emerge el significado de la historia cultural de entre los procesos de su significación (1981: 108).

Los 'eventos' a los que se hace referencia aquí son los que suelen denominarse el Romanticismo y la Ilustración, movimientos literarios que se desarrollan dentro del contexto de los siglos XVIII y comienzos del XIX en las letras inglesas - este último en cuanto al Romanticismo -, y que, asimismo, corresponden a dos tendencias más generales dentro de la historia cultural que marca el período en cuestión. Dicho así, se evitan aquí las denominaciones más tradicionales, dentro del campo de la historia literaria inglesa, de neoclasicismo, o era augusta (Augustan Age) para referirse a la parte del XVIII que no corresponde al Romanticismo. Así preferimos referirnos a este mismo periodo, basándonos en Watt (1957) y Hunter (1990), con relación al auge de la novela como forma literaria que influye sobre las letras europeas, incluyendo la literatura inglesa, contribuyendo al proceso, aunque en sus inicios, de la democratización cultural, relacionada con la figura del individuo, que constituye uno de los rasgos principales de la Ilustración, como nos recuerdan Hulme y Jordanova al hablar de los autores de novelas de viaje: "They saw themselves as metaphorical 'travellers'; concerned -as real travellers must be- with firsthand experience, scepticism towards written authorities, and the production of knowledge acquired in a rigorously empirical way. This is one facet of the current of radical individualism that has been deemed central to the Enlightenment" (1990:12).

Al llevar el análisis de la historia cultural hasta la postmodernidad, en cuanto a su relación con el desarrollo de la literatura inglesa, se pretende alcanzar el objetivo principal de este estudio que consiste en señalar de qué modo, a raíz de la contribución de la Ilustración y el Romanticismo a dicho desarrollo, la postmodernidad como impasse puede ser reactivada en términos histórico-temporales. Un resumen - siempre inadecuado - de la 
teoría de la cultura postmoderna como impasse propuesta por Jean-François Lyotard en The Postmodern Condition, señalaría cómo el filósofo francés traza el gráfico del reemplazamiento de las grandes narrativas culturales del Humanismo y de la Ilustración por las de la Ciencia y la Tecnología, haciendo que la auto-perpetuación metodológica ('performativity,' en inglés) llegue a ser el factor que domina el modo de pensar de las sociedades avanzadas (1984: 46). Lo que argumentamos aquí es que el Romanticismo y la Ilustración, a través del auge de la Novela como forma cultural durante el siglo XVIII, inauguran, dentro de la consolidación de la modernidad, la era de los discursos totalizadores. Asimismo, dicho proceso se manifiesta por medio de metáforas estructurales y modelos teóricos basados en lo espacial.

Además, cualquier reactivación del carácter ahistórico de la postmodernidad tendría que tener como referente histórico-cultural el hecho de que, conjuntamente, los dos movimientos literarios que nos conciernen adquieren su identidad original gracias a la forma en la que nos permiten calibrar la transición entre un modo de entender la historia cultural basado en lo temporal y otro basado en lo espacial. En este mismo sentido, cualquier planteamiento teórico acerca de la renovación histórica de la postmodernidad necesita basarse en el recuerdo de 'estructura' cultural del siglo XVIII que, en parte, se entiende en términos temporales o, dicho de otro modo, en términos que no implican lo espacial.

Ya, de por sí, a raíz de lo dicho anteriormente, se puede concebir en términos espaciales la relación entre 'estructura,' la Ilustración dentro de la historia cultural, y 'evento,' ésta presentada como fenómeno literario, siendo la figura de mise-en-abyme la más apropiada para poner de manifiesto dicha relación interactiva. El otro objetivo de este estudio, pues, es presentar un análisis de extractos de obras literarias representativas para así poder aproximarse a una descripción de la supresión de lo temporal en la mentalidad cultural del siglo XVIII, señalando al mismo tiempo que, irónicamente, esta misma supresión permite advertir cómo la visión temporal de la realidad se convierte en un mecanismo liberador que llega a la superficie durante la etapa postmodernista de la literatura inglesa, y dentro de la postmodernidad.

Se podría argumentar, en cierto sentido al menos, que la supresión del tiempo como punto de referencia cultural en el siglo XVIII queda vinculada al empequeñecimiento del individuo frente al universalismo que caracteriza a todos los individuos, desde la perspectiva del Romanticismo, y otro proceso semejante que afecta al individuo en un sentido reduccionista al someterle a las leyes estéticas de su representación en la novela como forma. Para poder explorar el papel liberalizador de los movimientos culturales del Romanticismo y de la Ilustración, identificada ésta con el auge de la Novela, dentro del contexto más amplio, o 'estructura,' de la historia cultural que culmina en la postmodernidad, dichos movimientos tienen que negativizarse en términos del contexto histórico del XVIII y XIX. De esta forma, se ofrece aquí una visión alternativa, de contextualización histórica más amplia, a la interpretación transcendentalista y triunfalista del Romanticismo por parte de la crítica de la llamada 'escuela arquetípica' de los años 40 y 50, representada por obras como The Romantic Imagination (1950), de C. M. Bowra, y The Mirror and the Lamp (1953), de M. H. Abrams. Y, asimismo, nos alineamos con aquella corriente crítica, dentro del campo de los estudios de la evolución de la Novela 
inglesa, que adopta una actitud ecuménica ante el tema de los discursos que preceden a ésta y que la alimentan, una corriente que se va consolidando en los ochenta a través de estudios como los de J. Paul Hunter (1979), James Sambrook (1986) y John F. Tinkler (1988).

En realidad, pues, se propone el socavamiento del idealismo utópico que contribuye a definir la Ilustración. Además, en este mismo sentido, resulta equivocado identificar al individuo social con el ser esencial, o el 'yo' ('self' en inglés), aunque pudiera parecer que exista un vínculo entre este ser y el fenómeno del tiempo, tal y como lo resalta Georges Poulet al describir el concepto de 'momento': "It is the greatness of the eighteenth century to have conceived the prime moment of consciousness as a generating moment and generative not only of other moments but also of a self which takes shape by and through the means of these very moments" (1956: 23).

El verbo 'to shape,' sugiriendo 'formar' o 'esculpir' en español, subraya cómo queda identificado el 'yo' esencial de la cultura de la Ilustración con lo espacial a diferencia de lo temporal. Al mismo tiempo, merece recordar que este mismo 'yo,' como producto de dicha cultura en su vertiente utópico-idealista, está vinculado a un proceso espacial de dimensionalización que puede identificarse con la visión histórica de Kant que entiende el proyecto del perfeccionamiento del Hombre en función del perfeccionamiento de la especie, un proceso que se deja de medir en términos temporales, mientras que se calibra en términos evolutivos. Gulliver, en la novela de Swift de 1726 Gulliver's Travels, evoluciona dentro de un proceso dimensionalizador por medio de una concienciación cada vez más matizada y calibrada en el espacio experiencial, basada en el reciclaje acumulativo de experiencias debido a múltiples operaciones comparativas derivadas del conocimiento, cada vez más matizada / calibrada en el espacio experiencial, de un gran número de sociedades.

A raíz de lo que se acaba de indicar, pues, el 'momento,' como unidad de experiencia matizable en términos espaciales, no sólo queda registrado culturalmente con arreglo al idealismo utópico de la Ilustración con relación al perfeccionamiento de la humanidad, sino también en función de la precariedad del individuo que empieza a operar dentro del ambiente capitalista de la modernidad. La trayectoria picaresca de la protagonista de Moll Flanders, la novela de Defoe de 1722, sirve de ejemplo de cómo el 'momento' pone al individuo al filo de la navaja constantemente -en el caso de Moll, en cuanto a lo sentimental al igual que a lo laboral-profesional.

Teniendo en cuenta el planteamiento original de este estudio, a través del cual se contempla la historia cultural en términos textuales, la oscilación estroboscópica entre el idealismo y la precariedad constituye un intertexto, lo que Louis Montrose denomina 'ironía intertextual' en otro contexto, es decir su estudio histórico-cultural del teatro de Shakespeare y la política isabelina (1992: 121). Este mismo intertexto confirma que el individuo social de la modernidad ya está sometido a la presión de los sistemas, esos sistemas que atrapan y pulverizan a los seres humanos tal y como queda reflejado en la poesía de William Blake a finales del siglo XVIII y las novelas de Charles Dickens en la franja central de la era victoriana, 'the mid-Victorian Age'.

Al volver a la novela Moll Flanders de Defoe, el 'intertexto irónico' relacionado con la Ilustración no sólo resalta paradigmáticamente la precariedad que marca la existencia del individuo social tal y como queda representado en la nueva forma literaria de la Novela, 
sino también, refiriéndonos a Lyotard, el auge de ésta como 'gran recit'. En este caso, como puede ejemplificarse por medio del episodio en el que Moll comete su primer robo, el 'intertexto irónico' tiene su paralelo en el subterfugio que permite a la Novela absorber para sí cualquier otra clase de discurso. Además, este mismo proceso constituye una ironía metaliteraria, ya que, como demuestra magistralmente J. Paul Hunter en Before Novels: The Cultural Contexts of Eighteenth-Century English Fiction (1990), la Novela como discurso emerge de entre múltiples otros discursos a lo largo del XVIII. El proceso conlleva la transformación de una dependencia total en un dominio absoluto.

En cuanto al extracto de Moll Flanders que se cita a continuación, el 'intertexto irónico' y metaliterario revela cómo el arte de la narrativa se apodera del discurso del arte del delineante a través de referencias a ángulos, planos y perspectivas, un proceso que se define en términos espaciales:

Wandring thus about I knew not whither, I pass'd by an Apothecary's Shop in Leadenhall Street, where I saw lye on a Stool just before the Counter a little Bundle wrapt in a white Cloth; beyond it stood a Maid Servant with her Back to it, looking up towards the top of the Shop; where the Apothecary's Apprentice, as I suppose, was standing up on the Counter, with his Back also to the Door, and a Candle in his Hand, looking and reaching up to the upper Shelf for something he wanted so that both were engag'd mighty earnestly, and no Body else in the Shop.

This was the Bait; and the Devil who I said laid the Snare, as readily prompted me, as if he had spoke, for I remember, and shall never forget it, 'twas like a Voice spoken to me over my Shoulder, take the Bundle; be quick; do it this Moment... (DEFOE, 1968: 191)

Tanto en el caso del episodio narrado, como en cuanto a la evolución de la Novela como forma, lo picaresco se ha profesionalizado. Este fenómeno de la profesionalización, junto con la consolidación de la especialización en mucho campos, constituye uno de los rasgos más destacados del periodo de la Ilustración, como nos recuerda Lyotard a lo largo de The Postmodern Condition. En este sentido, es el arte de hacer novelas el que adquiere importancia en el siglo XVIII de tal forma que cualquier novela específica carece de importancia; el arte de narrar termina sistematizándose.

Dicho así, corolariamente hablando, el cambio que pudiera realizarse para así contrarrestar el poder de los sistemas sería de naturaleza histórica y, por tanto, se entendería al individuo histórico capaz de llevar a cabo semejante hazaña en términos temporales. Al igual que el individuo se encuentra sometido al poder del sistema socio-capitalista en el XIX, la novela como texto individual queda absorbido por la Novela como forma literaria, habiendo emergido a lo largo del siglo XVIII de entre múltiples otros discursos, proceso este último que ha sido descrito magistralmente por Hunter, como ya se ha mencionado. Además, la relación individuo-sistema, novela-Novela queda constituida en función del modelo espacial del mise-en-abyme. Asimismo, se comprende la representación del individuo en la novela inglesa entre los siglos XVIII y XIX con arreglo a dicho modelo, ya que el personaje individual siempre queda trazado con respecto a cómo se calibra el grado de su individualidad frente a los antecedentes prototípicos que le predeterminan. Los victorianos no habrían entendido la novela con relación a su autor, es decir con arreglo al novelista individual, sino como un género de consumo, como indica Alan Horsman en su 
estudio de métodos de publicación durante el siglo XIX en el primer capítulo de The Victorian Novel (1990: 1-11).

Por lo tanto, al principio de este estudio, se plantea cualquier el fenómeno que permite ir más allá de la postmodernidad, en cuanto a la historia cultural se refiere, como el equivalente de un hecho histórico relacionado con lo individual, sea poeta o sea novelista, sobre todo porque nos permite volver a entender la cultura con relación a lo temporal. A nuestro parecer, en el plano de la historia literaria, el hecho histórico en cuestión sería el emerger de la Novela(-como-novela) frente a la novela postmodernista durante la última década del siglo XX. Este fenómeno permite concebir, al menos imaginativamente, una etapa histórica que no está dominada por el signo de dicha postmodernidad.

Volviendo al modelo espacial del mise-en-abyme, como tal modelo encierra un proceso de tensión o incluso lucha histórica, como indica la propia distribución de sus dos componentes desiguales. Dicha lucha, se podría afirmar, al menos tentativamente, queda vinculada a la ya mencionada precariedad asociada con la supervivencia dentro de la modernidad, mientras que la conciencia del individuo moderno percibe dicha precariedad en función de lo espacial. En este sentido, adquieren relevancia las palabras de Poulet al referirse al individuo cartesiano del siglo XVII como la figura, la del "cogito ergo...," que contribuye a la consolidación definitiva de la modernidad:

Human thought no longer feels itself a part of things. It distinguishes itself from them in order to reflect upon them, and thus is no longer upheld by their own power of enduring... Separated from the duration of things, and even from that of the modes of its existence, the human consciousness finds itself reduced to existence without duration. It is always of the present moment (POULET, 1956: 13).

Entender el tiempo como la constante inevitabilidad del momento presente implica prepararse para la etapa cultural de la postmodernidad que percibe el tiempo como el equivalente de la instantaneidad. En ambos casos el tiempo llega a equivaler al espacio y, como nos indica Poulet, el individuo cartesiano queda identificado con un espacio fuera del espacio general de la cultura, constituyendo, si se retoma la ya mencionada terminología propia del campo de la historia cultural, un 'evento' con relación a la 'estructura' del periodo cultural en cuestión, teniendo en cuenta que dicha relación también puede representarse por medio de la figura del mise-en-abyme.

En cuanto al individuo cartesiano del siglo XVII, todavía se manifiesta la cuestión de la supervivencia en términos menos alarmantes que en la época de la Ilustración y el XIX cuando se convierte en una lucha propia de la modernidad. El individuo del XVII establece una equivalencia entre dicha necesidad de sobrevivir y la posibilidad de renacer en cada momento, como indica Poulet: "[T]he Cartesian mind must, in each separate moment, recapture the evidence of truth" (1956: 17).

Al recoger la idea, señalada más arriba por Poulet, de la separación de la 'duración' de las cosas que experimenta la conciencia humana a partir del XVII, se descubre que, desde una perspectiva metaliteraria, el poeta inglés del Romanticismo padece dicha separación por no encontrarse capacitado como artista individual para romper la incorporación enigmática a lo eterno perpetuada dentro de la estética de este mismo movimiento literario. En este sentido, la 'duración,' al llegar a equivaler a lo eterno, se transforma en un 
fenómeno espacial. Además, aunque el poeta individual se encuentre en espacio aparte, que implica el establecimiento de nuevo de un modelo estético basado en la figura del mise-enabyme, el efecto totalizador de lo eterno en términos espaciales hace que cualquier alternativa adquiera un carácter temporal.

La absorción por parte de la estética romántica de lo eterno de cualquier poema resalta cómo éstos se reproducen, otra vez en términos metaliterarios, como objetos que se interrogan acerca de su propia naturaleza enigmática. Así que, en el caso del artefacto que protagoniza la oda de Keats, titulada "Ode on a Grecian Urn" (1819), la urna misma constituye un enigma de la eternidad:

Thou still unravish'd bride of quietness,

Thou foster-child of silence and slow time,

Sylvan historian, who canst thus express

A flowery tale more sweetly than our rhyme-

What leaf-fringed legend haunts about thy shape

Of deities or mortals, or of both,

In Tempe or the dales of Arcady?

(Gittings, 1966: 127)

De modo enigmático queda establecida una síntesis entre la naturaleza como medio y el tema de la interacción de ésta y el arte, fenómeno que hipnotiza al poeta (voz de la composición), como indican las oraciones interrogativas que, paradójicamente, él mismo compone pero sin ser consciente del carácter inconsciente del proceso. Dicha incapacidad le sitúa, pues, en una dimensión temporal. Por este motivo, al final del poema, sólo parece que el poeta habla desde dentro de la dimensión de lo enigmático, con voz de oráculo, dado el tono bíblico de los versos: "Beauty is truth, truth beauty, - that is all / Ye know on earth, and all ye need to know."

Al mismo tiempo, sin embargo, dicho pronunciamiento tiene que contrastarse con la siguiente exclamación, asimismo dirigida a la urna, que aparece en esta misma última estrofa: "Thou, silent form, dost tease us out of thought / As doth eternity: Cold Pastoral!" En cuanto a su identidad como acto de elocución, "Cold Pastoral!," por un lado, puede ser considerada un acto expresivo, sugiriendo admiración, incluso adoración, hacia la naturaleza enigmática del artefacto griego, y, por otro, un acto comisivo, indicando un gesto de rechazo vinculado con un sentimiento de frustración. De hecho, la emoción inicial que impregna el poema termina transformándose en frustración y confirmando así el modo en el que el proceso creativo siempre queda preso del enigma de lo eterno.

También se juntan la fascinación y la admiración en las formas vocativas que emergen en la ya citada primera estrofa donde se compara la naturaleza enigmática de la urna como obra de arte con la condición de la virginidad. Asimismo, en estos primeros versos se describen los orígenes de la urna en términos igualmente misteriosos, asociados éstos tanto con lo revelador como con lo emergente, constituyendo su esencia una síntesis de lo natural y lo artístico, por un lado, y, al mismo tiempo, de lo social y lo espiritual.

El aumento en el grado de fascinación queda reflejado, dentro de la estrofa, con la ya mencionada transición entre lo vocativo y lo interrogativo mientras que, en la medida en la que el poema avanza, esta misma indefinición empieza a adquirir connotaciones negativas 
en relación con la capacidad del poeta, y del lector, de sintonizar con el arte verdadero. Dicho de otro modo, el Arte, como fenómeno asociado con lo eterno, constituye un catalizador de la melancolía que acosa al poeta. De este modo, el poeta queda fuera del proceso centrípeto y exigente de la estética del Romanticismo, un proceso que contrasta con la dinámica dimensionalizadora que caracteriza a la estética de los textos relacionados con la Ilustración, y que se configura como centrífugo.

También puede ejemplificarse este mismo grado de negatividad en cuanto a la relación del poeta individual con la estética romántica al referirse a "Tintern Abbey," de Wordsworth, compuesto en 1798. Aquí un esquema temporal supuestamente expansivo termina contrayéndose. Con arreglo a la posibilidad de nutrirse espiritualmente del paisaje del río Wye y del emplazamiento de la abadía cisterciense a orillas del mismo, el relevo generacional que enmarca dicho proceso queda teñido por una sensación de derrota.

En los primeros siete versos el poeta se dirige a su hermana más joven del siguiente modo:

Five years have passed; five summers with the length

Of five long winters! And again I hear

These waters, rolling from the mountain springs

With a sweet inland murmur. Once again

Do I behold these steep and lofty cliffs,

Which on a wild secluded scene impress

Thoughts of more deep seclusion, and connect

The landscape with the quiet of the sky.

(WU, 1994: 240)

La demora en la aparición de la unidad sintáctica de coordinación final indica una mezcla de modestia y preocupación sincera por su hermana por parte de la voz del hermano mayor, cerca ya éste de la muerte. No obstante, el legado verdadero que llega hasta la hermana queda constituido por la mera exhortación que, supuestamente, ella, a su vez, legará a la generación que le seguirá. Al final resulta ser un legado de incapacidad, quedando constancia así de cómo el poder enigmático de la muerte y de la naturaleza triunfará sobre la felicidad compartida por los dos hermanos ante el paisaje de Tintern. Como ocurre en "Ode on a Grecian Urn" de Keats, el optimismo inicial termina situándose dentro de parámetros negativos.

Teniendo en cuenta dicha negatividad, el poeta hace referencia a la naturaleza de la siguiente forma:

'tis her privilege,

Through all the years of this our life, to lead

From joy to joy. For she can so inform

The mind that is within us, so impress

With quietness and beauty, and so feed

With lofty thoughts, that neither evil tongues, 
Al deconstruir el término "privilege," ya queda confirmado el sentido de 'elevación,' acompañado por la acepción de 'distanciamiento' (incluso de 'desprecio'), que encierra el término léxico "lofty". De este mismo modo, queda subrayado la represión espacial de los hermanos con arreglo a sus vínculos con el poder generativo de la naturaleza. Dicho así, resulta que sólo la fé, un fenómeno de poca envergadura, sostiene las exhortaciones del poeta dirigidas a su hermana. Tanto éstas como la existencia de los hermanos puede considerarse efímeras.

Incluso en el poema "The Tiger" (1794) de William Blake, interpretado tradicionalmente como una celebración de las fuerzas míticas de la naturaleza y el universo, vuelve a confirmarse la imposibilidad de la creatividad verdadera por parte del poeta individual:

Tiger, tiger, burning bright

In the forests of the night,

What immortal hand or eye

Could frame thy fearful symmetry?

(KEYNES, 1966: 214)

El distanciamiento temporal sugerido por el calificativo "immortal" hace aún más patente la frustración implícita que caracteriza a la voz del poeta, ya que no solamente le parece imposible a éste aprovechar el poder de lo mítico, sino también se siente incapaz de desprenderse del eterno recuerdo de dicha imposibilidad, tal y como enfatiza el sintagma "burning bright". Así que, el éxtasis se hace permanentemente agónico.

La lucha para evadir el agotamiento artístico y espiritual también emerge como el tema implícito del poema de Shelley titulado "Ode to the West Wind" (1820), donde otra nota de desesperación socava la supuesta glorificación del poder de la Poesía, personificada por el viento, transformándola en una dependencia que casi puede ser interpretado como sinónima de esclavitud: "Oh wind, / If winter comes, can spring be far behind?" (WU, 1994: 873). De nuevo, contemplar un posible proceso de renovación revela un estado de agotamiento creativo.

Por este último motivo, la composición de Shelley constituye un acto desesperado de creatividad, mientras que la precariedad que marca su desarrollo puede expresarse en términos temporales como una cuestión de vida o muerte, como la interacción de la extinción y la supervivencia: "If I were a dead leaf thou mightest bear; / If I were a swift cloud to fly with thee" (872)

Además, la naturaleza paradójica de la creatividad misma, que consiste en la necesidad de buscar la inspiración con el fin de dar expresión a dicha búsqueda, nos recuerda la inevitabilidad de convivir permanentemente con la clase de imposibilidad ya explorada con arreglo a "The Tiger" de Blake o la composición de Keats que gira alrededor de la urna griega. La presencia perenne de esta misma imposibilidad queda definida por Keats como 'capacidad negativa' ("negative capability") en una carta escrita en diciembre de 1817 (GITTINGS, 1966: 40-41). En este sentido, se advierte un tono casi de masoquismo en el 
poema de Shelley: "Drive my dead thoughts over the universe / Like withered leaves to quicken a new birth" (872).

En estos versos finales del poema, el símil que irrumpe en la cadena sintáctica, señalando la síntesis de símbolos relacionados con la inspiración, confirma el tono de desesperación en la voz del poeta, al igual que su dependencia frente a las fuerzas mágicas a las que hacen alusión dichos versos:

And, by the incantation of this verse,

Scatter, as from an unextinguished hearth

Ashes and sparks, my words among mankind!

Be through my lips to unawakened earth

The trumpet of a prophecy! (872-3)

Es como si la voz poética fuera obligada a emplear dos símbolos simultáneamente, confirmándose así como signo de debilidad, mientras que, de modo paralelo, una interacción igualmente estroboscópica configura la relación entre la voz del poema y su fuente de inspiración, el viento del oeste. De esta forma, vuelve a subrayarse la desesperación que caracteriza a dicha voz: "Be thou, spirit fierce, / My spirit! Be thou me, impetuous one!" (872).

Incluso, se puede detectar una nota de humor en la posible reacción negativa de la tierra como consecuencia de ser despertada por 'la trompeta de la profecía'. Así, pues, la impetuosidad artística y la anti-naturalidad creativa quedan vinculadas con el fracaso en el quehacer poético. En términos temporales, además, el futuro de la poesía está en juego, como refleja el último verso ya citado al referirse a la primavera como momento de la reanudación de la agonía del poeta individual a diferencia de la renovación de sus poderes de creatividad.

En "Kubla Khan: A Vision," compuesto por Coleridge en 1816, se advierte la existencia de una situación semejante a la que se produce en el poema de Shelley. La naturaleza hiperbólica de la descripción del poeta como 'magus,' junto con el deslumbramiento que acompaña cualquier intento de mirarle a los ojos, contribuye a la creciente desconfianza del lector ante la credibilidad de aquél que se va convirtiendo en una figura jocosa:

And all should cry, 'Beware, beware!

His flashing eyes, his floating hair!

Weave a circle round him thrice,

And close your eyes with holy dread -

For he on honey-dew hath fed

And drank the milk of paradise.'

(WU, 1994: 515)

En términos temporales, que conlleva la acepción de lo eterno desde la perspectiva del dominio de la Poesía sobre cualquier poeta individual dentro del Romanticismo, el peligro que corre éste es seguir siendo ridiculizado por las generaciones venideras. En este sentido, la versión deconstruida del tiempo verbal futuro-hipotético ("all should cry") socava la idea de asombro, reemplazándola con otra que conlleva la acepción de burla. 
En el ensayo teórico "A Defence of Poetry" (1821), de Shelley, se encuentra otro ejemplo de cómo parece detectar el socavamiento de la idealización y la mitificación tradicionales que marca el discurso del Romanticismo en la poesía inglesa. Lo impredecible que resulta ser la poesía, con sus vaivenes casi caprichosos en cuanto a lo temporal, constituye el mecanismo de exclusión que destierra al poeta individual: "[T]he mind in creation is as a fading coal which some invisible influence, like an inconstant wind, awakens to transitory brightness; this power arises from within, like the colour of a flower which fades and changes as it is developed, and the conscious portions of our natures are unprophetic either of its approach or its departure" (BLOOM, 1966: 443). Aquí lo jocoso se manifiesta por medio de la auto-parodia como queda subrayado por le referencia a lo profético, dado que, tradicionalmente dentro de la estética del Romanticismo, se conciben como sinónimas las figuras del poeta y el profeta. Además, el modo inconexo y arbitrario en el que la poesía queda activada vuelve a resaltar cómo el tiempo no se considera un factor esencial en dicha estética y, precisamente por este motivo, la Poesía no puede ser identificada con lo temporal.

Teniendo en cuenta lo dicho anteriormente, cualquier momento equivale a toda la Poesía en su totalidad, tal y como se desprende de Milton de Blake:

\author{
For in this Period the Poet's Work Done: and all \\ the Great \\ Events of Time start forth \& are conceived in such a \\ Period, \\ Within a Moment, a Pulsation of the Artery.
}

(KEYNES, 1966: 490)

Dicho de otra forma, cada momento opera dentro de una escala mítica y la responsabilidad que conlleva ser digno de esta misma herencia mítico-universal dota al discurso poético del Romanticismo inglés de una carga negativa que implica un estado de precariedad para el poeta individual que se culmina en la eliminación de este del espacio mítico en cuestión. Así que éste queda identificado con lo temporal.

Como se deriva del ensayo de Shelley que nos concierne aquí, irónicamente el purgar de lo familiar coincide con el acto creativo que incluye, además, el purgar del poeta individual. Éste, dado el carácter mítico de la Poesía, llega a ser indigno de dicha estética, sobre todo porque está atrapado en el tiempo histórico:

[Poetry] makes us the inhabitants of a world to which the familiar world is chaos. It reproduces the common universe of which we are portions and percipients, and it purges from our inward sight the film of familiarity which obscures from us the wonder of our being. It compels us to feel that which we perceive, and to imagine that which we know. It creates a new universe, after it has been annihilated in our minds by the recurrence of impressions blunted by reiteration. (445)

El nuevo universo que crea el arte poético emerge como consecuencia de un proceso cataclísmico, como indica el empleo del verbo "annihilate," mientras que esta dimensión supuestamente renovada queda identificada con lo paradisíaco y, por lo tanto, no puede ser poblada. Incluso la dimensión de lo sublime, tan alabada por el Romanticismo, queda fuera 
de lo que pudiera considerarse la normalidad de lo temporal, ya que ésta también se desfamiliariza por medio de la estética absorbente de la exclusividad del espacio romántico, como indica la referencia a "momentary" a continuación: "In its more energetic rendition the sublime is a kind of homeopathic therapy, a cure of uneasiness by means of the stronger, more concentrated -but momentary- anxiety involved in astonishment and terror" (Wu 46).

De igual modo se advierte un alto grado de tanto idealismo como perfección con arreglo al medio estético de la Poesía en el ensayo de Thomas de Quincey, titulado "On the Knocking at the Door in Macbeth" (1823): "Oh mighty poet! Thy works are not those of other men .... [I]n them there can be no too much or too little, nothing useless or inert, but that the further we press in our discoveries, the more we shall see proofs of design and selfsupporting arrangement where the careless eye had seen nothing but accident" (WU, 1994: 686). Tanto el diseño misterioso, que está escondido, como la figura del poeta mítico, contribuyen al proceso de erosión a la que la figura del poeta individual está sometida, quedando identificado éste con "the careless eye," en el texto escrito por de Quincey y "the film of familiarity," en cuanto al texto teórico ya citado de Shelley. El calor humano, pues, asociado con la individualidad, queda eliminado, como si de un proceso de desnaturalización se tratase. De ahí viene la ironía que se puede detectar en las palabras de Wordsworth en el prefacio que escribe a su colección de "Lyrical Ballads" en 1802, una ironía derivada de la deconstrucción de su discurso: "The principal object, then, which I proposed to myself in these poems, was to choose incidents and situations from common life....and to make these incidents and situations interesting by tracing in them the primary laws of our nature..." De este modo, el poeta pretende ofrecernos lo siguiente: “...a selection of language really used by men" (WU, 1994: 252).

En cuanto al extracto que se acaba de citar, se puede establecer una equivalencia, a efectos de la deconstrucción del discurso de Wordsworth, entre la el uso que el poeta inglés hace del término, y la idea dimensión de lo real en relación con la operatividad del lenguaje en el medio textual tal y como la desarrolla Lacan en su teoría psicoanalítica, recogida en parte, claro está, en The Language of the Self (1968) que el post-estructuralismo ha aprovechado para explicar el acto interpretativo. Teniendo en cuenta que Lacan asocia la dimensión real del lenguaje con la experiencia misma de éste como medio, se descubre la presencia del mismo grado de idealización del lenguaje en las ideas del filósofo francés que en las del poeta romántico inglés. Dicho así, pues, el lenguaje 'real' en el que hace hincapié Wordsworth resulta ser la representación idealizada del lenguaje, mientras que tal idealismo implica el rechazo hacia lo socialmente real. En este mismo sentido, Wordsworth también confunde el individualismo social con la excepcionalidad inalcanzable propio del espacio excluyente del Romanticismo, como nos recuerda el ensayo titulado "The History in "Imagination"” de Alan Liu: "[T]he romantics reasserted nature's universal truth against that of history in the new form of individuality: the 'original' or transcendentally nonconventional self"'(1995: 92).

Inevitablemente, el discurso de Wordsworth ya queda incorporado al macro-discurso del Romanticismo, como si fuera una gran recit, basado en una estética de lo espacial que va en aumento en función de un proceso cada vez más dimensionalizador. Además, como nos recuerda Niall Lucy, por medio de una línea argumental que asocia tendencias 
renovadoras dentro del Romanticismo con el entrecruzamiento de la creatividad literaria y la teoría literaria dentro de la postmodernidad, lo productivo en cuanto a las composiciones de los poetas románticos queda vinculado con lo enigmático: "Their true nature is that their true nature, like the true nature of literature, remains forever out of reach of rational, scientific or pragmatic systems of calculation and knowledge. Their true nature, as exemplified by the nature of the literary text in general and the poem in particular, is that their nature is to be a question" (1997: 37). Sin embargo, lo enigmático como discurso envolvente, que, en parte, ha sido tema de este estudio, sigue siendo una zona estética exclusiva.

Mientras tanto, el punto culminante del proceso de espaciamiento que incide en la historia cultural europea, y anglo-americana, guarda relación con el Modernismo que distingue entre la conciencia superficial de la vida cotidiana y una conciencia más profunda de la experiencia inmediata, algo que se aprecia al mirar dentro de nosotros mismos de modo muy cuidadoso, algo que no suele hacerse nunca. De este modo, queda registrado de qué forma nuestros estados psíquicos se encuentran en un estado de flujo constante y así queda alterado el mismo objeto, visto por segunda vez, por el mero hecho de haberse visto anteriormente. Todo lo dicho hasta ahora pretende constituir un resumen del concepto de la temporalidad dinámica de la experiencia psíquica propuesto por Bergson en 1889, por medio del concepto de durée réelle, tal y como lo explica en su Essai sur les données immédiates de la conscience, traducido al inglés con el título de Time and Free Will, en 1910. Como indica el mismo Bergson en relación con la tendencia de engañarse en cuanto a la desvinculación que establecemos entre lo que percibimos y el modo en el que lo percibimos: "We instinctively tend to solidify our impressions in order to express them in language. Hence we confuse the feeling itself, which is a perpetual state of becoming, with its permanent external object, and especially with the word which expresses this object" (1992: 130).

Aunque Bergson pone énfasis en el papel del lenguaje como elemento que contribuye a la impresión de que la experiencia tiende a organizarse y solidificarse fácilmente, lo que queremos destacar es el papel del filósofo francés dentro de un movimiento intelectual de más amplio espectro, el cual influye sobre los escritores modernistas durante la transición entre los siglos XIX y XX, donde tienen cabida otras formas de designar la presentación de la realidad que existe debajo de las convenciones instrumentales que se utilizan para ordenarla: el "caos de sensaciones" de Nietzsche, la "inmediatez experiencial" de F.H. Bradley y la "corriente" o "stream of consciousness" de William James.

Se vuelve a advertir, pues, que el Modernismo, al igual que el Romanticismo y la Ilustración, a través de la estética de la Novela, tiende a universalizar lo que aparentemente queda manifestado como la exploración de la psique individual que corresponde a una historia (temporal, por tanto). Como afirma Bergson en la obra ya citada: "Now if duration is what we say, deep-seated psychic states are radically heterogeneous to each other, and it is impossible that any two of them should be quite alike, since they are two different moments of a life-story" (1992: 199).

Sin embargo, al igual que en el caso de la interacción de la estética de lo prototípico, por un lado, y de lo individual, por otro, que caracteriza el desarrollo de la novela inglesa del siglo XIX, a partir de la obra de Jane Austen, el proceso imaginativo que caracteriza a la 
estética del texto modernista se manifiesta por medio del tratamiento auto-complaciente de cualquier tema supuestamente específico o de carácter individualizado.

Para romper el encantamiento ejercido por el Postmodernidad como impasse, llega a ser necesario deconstruir el circuito cerrado en el que dicha etapa de la historia cultural ha quedado atrapada. Este mismo circuito, consistente en la perpetuación estroboscópica de lo relativo, ha sido descrito por Christopher Norris como la interacción de un discurso totalizador, que se asemeja a los del Romanticismo y la Novela dentro de la Ilustración, y otro de carácter múltiple que, por ser interactivo en cuanto al otro totalizador, también adquiere ese mismo carácter: "It is wrong to suppose that there must be some ultimate choice between, on the one hand, foundationalist theories that isolate truth from the context of discursive or linguistic justification, and on the other, the wholesale pragmatist view that truth is simply what counts as such according to our present, culture-specific aims and purposes" (1988: 81).

Al contrario del estado de impasse que las palabras de Norris siguen apoyando como raison d'être la postmodernidad, lo que pone de relieve el llamado 'debate' entre el ya mencionado Lyotard y el filósofo alemán, Jürgen Habermas, es que dicho modelo cultural sí puede ser deconstruido. Habermas tiende a criticar la filosofía postmoderna por dejar de lado las ventajas que se derivan de la aplicación de la razón. En este sentido, la segunda vertiente del 'debate' entre los dos filósofos, que gira alrededor de la teoría de la comunicación entre los seres humanos, quedando rechazada por Lyotard, es la defensa idealista que Habermas, autor del ensayo "Modernity - An Incomplete Project" (FOSTER, 1983: 3-15), hace del concepto del consenso en preferencia a una actitud más tolerante y abierta ante las múltiples posibilidades del discurso comunicativo. La interacción de estas posibilidades tiene su equivalencia dentro de la historia literaria reciente en el auge de muchas novelas que ya no pueden ser consideradas exclusivamente postmodernistas, novelas escritas por escritores como Penelope Lively, Eva Figes, Sebastian Faulks y Adam Thorpe, por citar algunos. Al considerarse como obras individuales, comparables entre sí, se dejan de concebir en función de un discurso totalizador como el Postmodernismo. Asimismo una aproximación crítica a la individualidad de dichas obras permite establecer una perspectiva en función de su valor histórico, ya que, al deconstruir la relación espacial, basado en la figura del mise-en-abyme, entre cualquier fenómeno y la globalidad de la cultura postmoderna. Así que, se contempla la posibilidad de volver a hablar de una tradición literaria, un fenómeno que atraviesa la dimensión espacial de la cultura de la postmodernidad, y que se entiende en términos histórico-temporales, al igual que lo hace $\mathrm{T}$. S. Eliot en su ensayo titulado "Tradition and the Individual Talent" (1919): "[T]he historical sense involves a perception, not only of the pastness of the past, but of its presence.... The historical sense, which is a sense of the timeless and of the temporal together, is what makes a writer traditional. And it is at the same what makes a writer most acutely conscious of his place in time, of his own contemporaneity" (1991).

La restitución de un modelo de la historia literaria basada en la interacción del artista individual y la Tradición, de naturaleza temporal, a diferencia de uno basado en una estética espacial envolvente, y por tanto excluyente, depende de la clase de exploración de dicha historia que se ha llevado a cabo en este estudio donde se ha destacado el papel de los 
movimientos del Romanticismo y la Ilustración, por medio de la Novela como forma emergente, en dicho proceso de renovación de la tradición literaria en lengua inglesa.

\section{BIBLIOGRAFÍA}

BERGSON, H. ([1889, 1910], 1960), Time and Free Will: An Essay on the Immediate Data of Consciousness, trad. F.L. Pogson, New York.

BLOOM, H., ed. (1966), Selected Poetry and Prose of Percy Bysshe Shelley, New York.

DEFOE, D. (1968), The History and Misfortunes of the Famous Moll Flanders, Harmondsworth.

DE LUCA, V.A. (1995), "Blake's Concept of the Sublime", Romanticism: A Critical Reader, ed. Duncan Wu, págs. 17-54, Oxford.

ELIOT, T.S. (1991), Selected Essays, London.

GITTINGS, R. (1966), Selected Poems and Letters of Keats, London.

HORSMAN, A. (1990), The Victorian Novel, Oxford.

HULME, P. / JORDANOVA, L., eds. (1990), "Introduction", The Enlightenment and its Shadows, págs. 1-15, London.

HUNT, L., ed. (1989), "History, Culture and Text”, The New Cultural History, págs. 122, Berkeley.

HUNTER, J.P. (1979), “The Insistent 'I'”, Novel, págs. 19-37.

----. (1990), Before Novels: The Cultural Contexts of Eighteenth-Century English Fiction, New York.

JAMESON, F. (1981), The Political Unconscious: Narrative as a Socially Symbolic Act, New York.

KEYNES, G., ed. (1966), Blake: Complete Writings, Oxford.

LACAN, J. (1968), The Language of the Self: The Function of Language in Psychoanalysis, trad. Anthony Wilden, Baltimore.

LIU, A. (1995), “The History in 'Imagination"”, Romanticism: A Critical Reader, ed. Duncan Wu, págs. 84-119, Oxford.

LUCY, N. (1997), Postmodern Literary Theory: An Introduction, Oxford.

LYOTARD, J. ([1979] 1984), The Postmodern Condition: A Report on Knowledge, trad. Geoff Bennington y Brian Massumi, Manchester.

MONTROSE, L. (1992), "A Midsummer Night's Dream and the Shaping Fantasies of Elizabethan Culture: Gender, Power, Form", New Historicism and Renaissance Drama, eds. Wilson R. y Dutton, R., págs 109-30, London. 
NORRIS, C. (1988), Deconstruction and the Interests of Theory, London.

POULET, G. (1956), Studies in Human Time, New York.

SAMBROOK, J. (1986), The Eighteenth Century: The Intellectual and Cultural Context of English Literature, 1700-1789, London.

TINKLER, J.F. (1988), "Humanist History and the English Novel in the Eighteenth Century”, Studies in Philology, págs. 510-37.

WATT, I. (1957), The Rise of the Novel: Studies in Defoe, Richardson and Fielding, Harmondsworth.

WU, D. (1994), Romanticism: An Anthology, Oxford. 
